\title{
Fluorescence Method for Studying Surface Orientation of Polymer Film Using Vacuum-Deposition Technique
}

\author{
Satoru OHMORI, Shinzaburo ITO, Yoshihiko ONOGI, \\ and Yasunori NisHIJIMA \\ Department of Polymer Chemistry, Faculty of Engineering, \\ Kyoto University, Sakyo-ku, Kyoto 606, Japan
}

(Received May 9, 1987)

\begin{abstract}
In order to establish a measurement for surface orientation of polymers, the fluorescence polarization method was applied for orientation analysis of fluorescent molecules vacuum-deposited on a polymer surface. The deposition behavior was also investigated. 1,6Diphenyl-1,3,5-hexatriene (DPH) was deposited on the surface of poly(vinyl chloride) (PVC) films. The orientation behavior of DPH on vacuum-deposition was examined by the polarization of fluorescence emitted from DPH. When a DPH molecule was introduced onto the surface of PVC films, the orientation was found to be three-dimensionally random. After these fluorescent films were drawn above $T_{\mathrm{g}}$, the surface orientation of the polymer chains was estimated by the dichroism of DPH, which was analyzed from the polarized components of fluorescence intensity. This system was compared with the DPH doped system in which DPH was distributed wholly in bulk. It was found that the orientation of DPH on the surface was slightly higher than that in the bulk. This slightly higher orientation in the polymer surface was also confirmed by birefringence measurements. The DPH was also vacuum-deposited onto some drawn PVC films. The orientation of DPH was higher on the film surface deposited after drawing than before drawing.
\end{abstract}

KEY WORDS Fluorescence / Surface / Orientation / Vacuum Deposition / Poly(vinyl chloride) / Elongation / Polarization /

The surface properties of polymers are important for application as functional materials, because the reaction and interaction with other substances often take place mainly at the surface. But the surface of polymers does not always show the same properties as the bulk. These differences mainly depend on the composition and structure. For the composition analysis of a polymer surface, a large number of investigations have been made by using various methods, for example, electron spectroscopy for chemical analysis (ESCA), attenuated total reflection IR spectroscopy (ATR IR), and secondary ion mass spectroscopy (SIMS).

In regard to the polymer structure, one of the most important factors is orientation, because polymers can change their properties continuously with the degree of their molecular orientation. The orientation of a polymer surface is especially important for the function of polymer materials. Recently there have been several studies on the orientation of polymer surfaces.

Sung et al. ${ }^{1,2}$ and Mirabella et al ${ }^{3,4}$ measured the surface orientation of polymers by the ATR IR method. According to Sung, uniaxially drawn polypropylene shows that the surface orientation is similar to the bulk. On the other hand, the surface of injection-molded polypropylene shows higher orientation than the bulk.

Wang et al. ${ }^{5}$ measured the surface orientation of polystyrene (PSt), which was co-extruded with polyethylene, by the methods of liquid contact angle and ATR IR. They reported that 


\section{S. OHMORI et al.}

the surface of PSt showed higher orientation than the bulk at the lower stage of extension.

In our laboratory, the molecular orientation of polymers was investigated by the fluorescence polarization method. ${ }^{6-8}$ In this method, fluorescent probes were dispersed in a polymer solid. By analyzing the polarized components of fluorescence emitted from the probes, one can get information about both the extent and type of polymer orientation. Since recent photon detectors are highly sensitive, this method should be easily applied for measuring the orientation in a small limited area, as long as the probes are introduced into the designed area. The vacuum-deposition technique has been investigated as a method for introducing molecules onto the surface of materials in various. fields. Vacuum-deposition of some molecules onto single crystals have been investigated. In these cases, it is said that a high vacuum system is necessary in order to attach molecules directly onto the surface. As to the case of a polymer, because the surface of a polymer film is very rough on a molecular scale and is considered thicker than $10 \mathrm{~nm}$, the molecules are easily attached to this surface. Then we deposited fluorescent probes onto polymer materials without very high vacuum treatment. In this report, in order to analyze the orientation of a polymer surface, the fluorescence polarization method was applied to the film introduced fluorescent probes by the vacuum-deposition technique.

By using this technique the following two facts could be clarified:

1) How is the rod-like fluorescent probe deposited onto the polymer surface?

2) Does the surface orientation differ from that of the bulk?

For the first point, the behavior of vacuumdeposition was observed by spectroscopic and microscopic measurements. The orientation of deposited probes was measured by the fluorescence polarization method. In order to confirm the evaluation of the experimental results, two kinds of model samples were measured for comparison. A surface-dyed PVA film and its highly drawn film were used for the model samples. The surface orientation was measured by this method for two kinds of polymers (PSt and PVC), on which 1,6-diphenyl1,3,5-hexatriene (DPH) was vacuum-deposited.

For the second point, in order to compare the orientation of a surface with that of bulk, DPH-doped PVC films were also prepared. The prepared samples were drawn and the orientation was estimated by absorption dichroism analyzed from the polarized components of fluorescence intensity.

Furthermore, for measuring the originally oriented surface, DPH was vacuum-deposited also on previously drawn PVC films, and then the orientation was measured by the same method.

\section{EXPERIMENTAL}

\section{Materials}

1,6-Diphenyl-1,3,5-hexatriene (DPH)

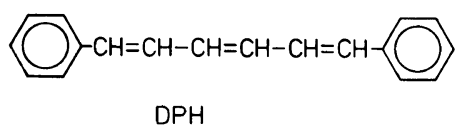

(Tokyo Chemical Industry Co., Ltd., Guaranteed Reagent) was chosen as a vacuum-deposition probe for surface orientation analysis. A stilbene derivative probe, whitex-RP 2 (Sumitomo Chemical Co., Ltd.)

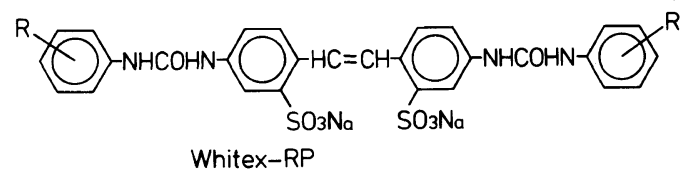

was used for dyeing poly(vinyl alcohol) films.

Poly(vinyl chloride) (PVC) was obtained from Nihon-carbide Co., Ltd. Poly(styrene) (PSt) and poly(vinyl alcohol) (PVA) were purchased from Nakarai Chemical Co., Ltd. These polymers were used as received.

\section{Preparation}

PVC films were cast from a $9 \% \mathrm{w} / \mathrm{v}$ tetrahy- 
drofuran (THF) solution at $40^{\circ} \mathrm{C}$. The obtained films were dried in vacuo in order to remove solvent thoroughly. The thickness of these films was about $250 \mu \mathrm{m}$.

PSt films were cast from a $10 \% \mathrm{w} / \mathrm{v}$ benzene solution at room temperature and PVA films were cast from a $9 \% \mathrm{w} / \mathrm{v}$ distilled water solution at $50^{\circ} \mathrm{C}$. After casting, these films were kept under reduced pressure for a day.

The elongation of sample films was carried out in a chamber whose temperature was regulated at $95 \pm 3^{\circ} \mathrm{C}$ for $\mathrm{PVC}$ and $90 \pm 3^{\circ} \mathrm{C}$ for PVA films. These temperatures are much higher than their glass transition temperatures, but sufficiently lower than the decomposition temperatures of these polymers. In the experiment to compare the orientation effects of a drawn sample to one undrawn, the same thermal treatment was given for the undrawn films. The PVC films were drawn in the range of $\%$-elongation from $0 \%$ to $400 \%$.

A separable reaction flask was used for vacuum-deposition. About $10 \mathrm{mg}$ of $\mathrm{DPH}$ were put on the bottom of the flask. Polymer films were held at about $10 \mathrm{~cm}$ above the bottom. The deposition was carried out under a pressure of $3-5 \mathrm{mmHg}$. The temperature of the flask was kept at $55^{\circ} \mathrm{C}$ by dipping in a water-bath. The deposition was continued for 3 - $8 \mathrm{~h}$; the deposition rate depended markedly on the kind of polymer. The deposition was continued to attach enough DPH for measurement onto the films. One side of the polymer film was washed with benzene in order to remove the deposited $\mathrm{DPH}$, and another side was used for measurement.

The quantity of deposited DPH was measured in the following way. A certain area was cut from the DPH deposited polymer films, and then dissolved in THF to measure the DPH fluorescence intensity. Comparing this value to fluorescence intensity of the THF solution whose DPH concentration was known, the absolute quantities of deposited DPH were calculated. The quantities were found to be a few $\mathrm{ng} \mathrm{cm}^{-2}$ for PVC films.
The surface-dyed PVA films were prepared by dipping the PVA cast films in a methanol solution of whitex-RP $\left(10^{-4} \mathrm{~mol} \mathrm{l}^{-1}\right)$ for an hour, and then by washing them in fresh methanol several times.

Doped films of PVC were prepared by dispersing the fluorescent molecules into the solution before casting. The DPH concentration in the obtained films was $3 \times 10^{-6} \mathrm{~mol} \mathrm{l}^{-1}$.

The following samples were prepared.

PVC 1) DPH doped films.

2) DPH vacuum-deposited films.

3) DPH vacuum-deposited, then drawn films.

4) drawn, then DPH vacuumdeposited films.

PSt 1) DPH vacuum-deposited films.

PVA 1) whitex-RP sufrace-dyed films.

2) whitex-RP surface-dyed, then drawn films.

\section{Measurements}

Fluorescence spectra were measured by a Hitachi model 850 fluorescence spectrophotometer. Absorption spectra were measured by a Shimadzu model UV-200S double beam spectrophotometer. Measurements of fluorescence decay were made by the single photon counting technique, using a PRA Inc., model 510B fluorescence lifetime instrument.

The apparatus for molecular orientation measurements is shown in Figure 1. Excitation light of suitable wavelength from a high pressure $\mathrm{Hg}$ lamp $(L)$ was selected by a filter $\left(F_{1}\right)$ (UV-D36A; Toshiba Co., Ltd.) and vertically polarized by a polarizer $(P)$. The sample film (S) was mounted on a rotatable holder designed to set films freely in plane or out of the plane of rotation. The fluorescence from the samples was observed at the angle $45^{\circ}$ to the propagation axis of excitation light. The polarized component was detected by a photomultiplier $\left(P M_{2}\right)$ through an analyzer $(A)$ and a cut-off filter $\left(F_{2}\right)$ (SC42; Fuji Film Co., Ltd.). The transmittance of these filters is shown in Figure 3. Intensity of the excitation light was 


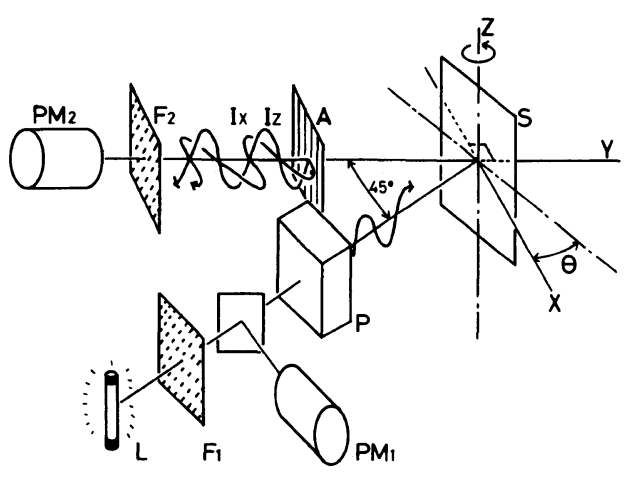

Figure 1. Optical system for measuring the polarized components of fluorescence intensity.

monitored by another photomultiplier $\left(P M_{1}\right)$, and relative intensity of the fluorescence to the excitation light was measured by a ratiometer. The orientation manner of the deposited probe on the polymer surface (whether the probes were oriented in three-dimensional (3D) random or two-dimensional (2D) plane-random) was measured by tilting the film as shown in Figure 1 . The tilting angle $\vartheta$ was varied from $0^{\circ}$ to $50^{\circ}$.

For the uniaxially deformed films, the orientation was measured by absorption dichroism of the probes, which was analyzed by the polarized components of fluorescence. The same apparatus (Figure 1) was used for measurement of dichroism. The film plane was fixed at right angles to the detector $\left(\vartheta=0^{\circ}\right)$, then rotated in the plane as shown in Figure 2. The intensities of fluorescence $I_{\mathrm{H}}\left(I_{X}+4 / 3 I_{Z}\right.$ at $\left.\gamma=90^{\circ}\right)$ and $I_{\mathrm{V}}\left(2 I_{X}+I_{Z}\right.$ at $\left.\gamma=0^{\circ}\right)$ were measured when the drawn axis was perpendicular and parallel to the plane of polarized excitation light, respectively.

The orientation of uniaxially drawn films was also measured by birefringence. An Abbe refractometer was used to obtain the birefringence of the film surface which was the difference in refractive indices between parallel and perpendicular directions to the drawn axis. The birefringence data for the bulk films can be calculated from the retardation using a

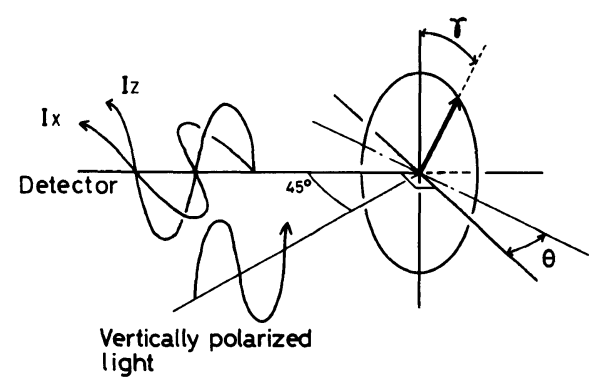

Figure 2. Angles specifying the orientation of the molecular axis (or drawn axis).

polarizing microscope (Nikon OPTIPHOTO). This microscope was also used to observe the penetration depth of probes from the polymer surface. The visible fluorescence of the probes was observed under UV-light irradiation.

\section{RESULTS AND DISCUSSION}

\section{Fluorescence Characteristics of Deposited Probes}

The absorption and fluorescence spectra of $\mathrm{DPH}$ in methylene chloride and in films are shown in Figure 3. The transmittance spectra of filters for measuring fluorescence polarization are also shown by broken lines in Figure 3. Since the spectra of another probe, whitex-RP, were in a similar range of the wavelength as DPH, the same filter system was used for whitex-RP.

The fluorescence spectra of the DPH doped films and DPH deposited films were almost the same as that observed in solution. Although DPH in films gives somewhat broader fluorescence spectrum compared with in solution, neither wavelength shift nor abnormal emission at longer wavelength is detected in their fluorescence spectra. Whether the DPH deposited films were drawn or not, the fluorescence lifetime did not change. As the fluorescent probes interact with each other in the highly concentrated state, their distribution can be known from the absorption and fluorescence spectra. From these results, we concluded that there is neither aggregation nor 


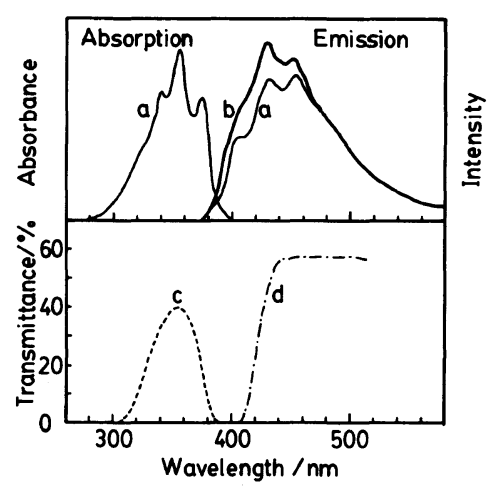

Figure 3. a) Absorption and fluorescence spectra of DPH in $\mathrm{CH}_{2} \mathrm{Cl}_{2}$, b) fluorescence spectrum of DPH doped in PVC, c) and d) transmittance of the filters: UV-D36A and two sheets of SC42.

crystallization of probes in the polymer films. As the quantity of deposited DPH in the surface region was too small to measure the absorption spectra by using the common spectrophotometer, the fluorescence intensity was used to evaluate the quantity of the probe per unit area of the surface. The quantity was a few $\mathrm{ng} \mathrm{cm}^{-2}$. The value, for example, of $3.4 \mathrm{ng}$ $\mathrm{cm}^{-2}$, means that the calculated absorbance for the film is only $1.5 \times 10^{-3}$ at maximum in the absorption spectrum, and that the average distance between the adjacent two probes would be $3.4 \mathrm{~nm}$ if the probes are only aligned as a monolayer.

As will be mentioned later, the orientation manner of deposited probes is 3D-random; that is, the probes become random on the surface. Microscopic measurements for these films under the irradiation of UV-light showed that the probes emitted fluorescence in the region less than a few $\mu \mathrm{m}$ from the surface. Since this observed depth was near the resolution of the microscope, we believe that the actual penetration depth of probes is much less than $1 \mu \mathrm{m}$.

The polarization character of DPH and whitex-RP is highly anisotropic. If the direction of the transition moment of fluorescence emission agrees with that of absorption, the anisotropy ratios of these probes in rigid matrices, in which the orientation of the probes is random, would be almost 0.4 . These anisotropy ratios were found to be 0.37 and 0.38 , for DPH in PVC and for whitex-RP in PVA, respectively. Because the shapes of these probes were very long, they were easily oriented along the orientation of polymer chains when the films were drawn.

\section{Orientation of Deposited Probes on Polymer Surfaces}

We investigated whether the orientation of the probes deposited on polymer was 3Drandom or 2D-plane-random in the film surface. This analysis was made by measuring polarized components of fluorescence intensity under linearly polarized light excitation. In Figure $1, I_{Z}$ and $I_{X}$ represent the polarized components of fluorescence, whose polarization axes are parallel to the $Z$-axis and $X$ axis, respectively. With rotating the sample films around the $Z$-axis (see Figure 1), $I_{Z}$ and $I_{X}$ were measured as a function of the angle $\vartheta$. The $\vartheta$ dependency of $I_{\mathrm{Z}}$ and $I_{\mathrm{X}}$ was considered for three kinds of orientation models.

Case 1: The probes are uniaxially oriented by an angle $\gamma$ from the $Z$-axis as shown in Figure 2. In this case the calculated values of intensity $I_{Z}$ and $I_{X}$ are

$$
\begin{aligned}
& I_{Z}=I_{0} \cos ^{4} \gamma \\
& I_{X}=I_{0} \cos ^{2} \gamma \sin ^{2} \gamma \cos ^{2} \vartheta .
\end{aligned}
$$

The ratio of these values is given by

$$
I_{X} / I_{Z}=\tan ^{2} \gamma \cos ^{2} \vartheta=K \cos ^{2} \vartheta
$$

where $K$ is a constant under the measurement of a fixed angle $\gamma$.

Case 2: The probes orient 2D-planerandomly on the film surface. $I_{Z}$ and $I_{X}$ are derived from integrating eq 1 and eq 2 from $\gamma=0$ to $2 \pi$. The derived values of $I_{Z}$ and $I_{X}$ are

$$
\begin{aligned}
I_{Z} & =\int_{0}^{2 \pi} I_{0} \cos ^{4} \gamma \mathrm{d} \gamma \\
& =(3 / 4) \pi I_{0}
\end{aligned}
$$




$$
\begin{aligned}
I_{X} & =\int_{0}^{2 \pi} I_{0} \cos ^{2} \gamma \sin ^{2} \gamma \cos ^{2} \vartheta \mathrm{d} \gamma \\
& =(1 / 4) \pi I_{0} \cos ^{2} \vartheta .
\end{aligned}
$$

The ratio of these values is given by

$$
I_{X} / I_{Z}=(1 / 3) \cos ^{2} \vartheta \text {. }
$$

Equations 3 and 6 show the same $\vartheta$ dependency of the ratio $I_{X} / I_{Z}$. If the transition moments of the probes orient parallel to the surface plane, the ratio $I_{X} / I_{Z}$ should be proportional to $\cos ^{2} \vartheta$. In order to distinguish between 2D-plane-random and uniaxial samples, the $\gamma$ dependence of $I_{X} / I_{Z}$ can be used according to eq 3 and 6 . However, the $\vartheta$ dependence of $I_{X} / I_{Z}$, being the same for both samples, the uniaxial samples can be used as model samples of 2D-plane-random orientation. The uniaxial sample which is set at an angle $\gamma=30^{\circ}$ is equivalent to the 2D-planerandom one. As we wanted to estimate only the $\vartheta$ dependency of the ratio $I_{X} / I_{Z}$, the sample was set at an angle $\gamma=45^{\circ}$ which is the best setting for precise measurement of the ratio $I_{X} / I_{Z}$.

Case 3: The probes orient 3D-randomly. $I_{Z}$ and $I_{X}$ are derived from integrating eq 1 and 2 from $\gamma=0$ to $\pi$ and from $\vartheta=0$ to $2 \pi$. The derived values of $I_{Z}$ and $I_{X}$ are

$$
\begin{aligned}
I_{Z} & =\int_{0}^{2 \pi} \int_{0}^{\pi} I_{0} \cos ^{4} \gamma \sin \gamma \mathrm{d} \gamma \mathrm{d} \vartheta \\
& =(4 / 5) \pi I_{0} \\
I_{X} & =\int_{0}^{2 \pi} \int_{0}^{\pi} I_{0} \cos ^{2} \gamma \sin ^{3} \gamma \cos ^{2} \vartheta \mathrm{d} \gamma \mathrm{d} \vartheta \\
& =(4 / 15) \pi I_{0}
\end{aligned}
$$

Then,

$$
I_{X} / I_{Z}=1 / 3=\text { constant. }
$$

In this case, the ratio $I_{X} / I_{Z}$ does not depend on the angle $\vartheta$. These calculated lines of $I_{X} / I_{Z}$ are shown in Figure 4, where the ratio is normalized to unity at $\vartheta=0^{\circ}$.

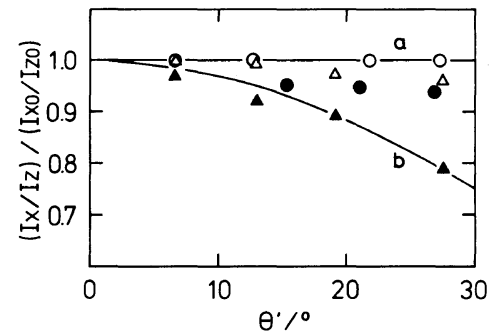

Figure 4. Dependence of $\left(I_{X} / I_{Z}\right) /\left(I_{X 0} / I_{Z 0}\right)$ on $\vartheta^{\prime}$, where $I_{Z 0}$ and $I_{X 0}$ are fluorescence components at $\vartheta^{\prime}=0^{\circ}$. Solid lines show theoretical values for a) 3D-random orientation, b) 2D-plane-random orientation and uniaxial orientation. Signs represent observed data for $(\triangle)$ PVA undrawn films, (A) PVA drawn films, (O) DPH deposited PVC films, and (O) DPH deposited PSt films.

There were two problems in the practical measurements. First, both the excitation light and fluorescence light refract at the interface between the polymer and air. This effect must be considered even if the probes are deposited or dyed on the surface of polymers, since the probes exist in the inner vicinity of the polymer surface. The angle $\vartheta$ in eq 2, 3, 5, and 6 should be rewritten by the angle $\vartheta^{\prime}$; the relation between $\vartheta$ which is set on the apparatus and $\vartheta^{\prime}$, which is an effective angle, is given as

$$
\sin \vartheta / \sin \vartheta^{\prime}=n \text {. }
$$

Here, $n$ is the refractive index of the sample. The other problem is birefringence of samples. Fortunately, our purpose being the measurement of probes in the surface region, the optical path length in films was so short that the effect of retardation was negligible.

Two PVA films were used as model samples. One sample film was obtained by dyeing the surface with a methanol solution of whitex-RP. This sample was expected to be 3D-random orientation. The other sample prepared by drawing uniaxially the dyed sample up to $300 \%$-elongation was expected to be uniaxial orientation. These films were set at an angle, $\gamma=45^{\circ}$. The results are plotted with triangles in Figure 4. The undrawn PVAwhitex-RP films show 3D-random orientation 
as expected. On the other hand, the drawn PVA-whitex-RP films show uniaxial orientation, which gives the same angle dependence with 2D-plane-random orientation. These results confirm the validity of this measurement.

The DPH-deposited films of PSt and PVC were then measured by this method. The results are plotted with solid and open circles, respectively, in Figure 4. The results of the PSt film include some experimental errors because of the weakness of the fluorescence intensity. However, the orientation of the deposited probes on the PSt film seems to be rather 3Drandom. On the other hand, probes on the PVC films showed perfect 3D-random orientation. For these two polymers, the DPH is deposited 3D-randomly by the vacuumdeposition.

\section{Surface Orientation of Drawn PVC}

The previous measurements indicated that the fluorescent probes deposited with 3Drandom orientation onto PVC surface. The fluorescence from the drawn films was analyzed for comparison of the orientation distribution of the probes in the surface and bulk. The deposited system gives an example for the orientation in the surface and the doped one gives that in the bulk. The undrawn samples were heated to the same temperature as the drawn ones, in order to make the experimental conditions the same. The orientation factor $f$ was evaluated by the absorption dichroism analyzed from the polarized components of fluorescence intensity,

$$
f=(3 / 2)\left\langle\cos ^{2} \omega\right\rangle-1 / 2
$$

where, $\omega$ is the orientation angle of the transition moment of the probe from the drawn axis $c$ as shown in Figure 5. Since the UVabsorption is too weak to observe the dichroism, the absorption dichroism was analyzed from the polarized components of fluorescence intensity using the apparatus shown in Figure 1. Total fluorescence intensity, which is proportional to absorbance, was

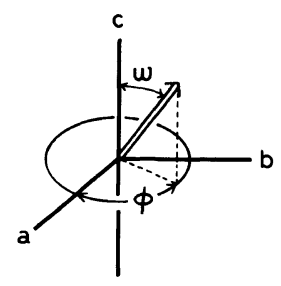

Figure 5. Orientation coordinate system for drawn films and angles specifying the orientation of the molecular axis.

calculated by the following equations

$$
\begin{aligned}
& I_{\mathrm{V}}=2 I_{X}+I_{Z} \\
& I_{\mathrm{H}}=I_{X}+4 / 3 I_{Z} .
\end{aligned}
$$

Where, $I_{\mathrm{V}}$ is the total fluorescence intensity when the drawn axis $\mathrm{c}$ is set at the position parallel to the polarization axis of the excitation light $\left(\vartheta=0^{\circ}, \gamma=0^{\circ}\right)$, and $I_{H}$ is the intensity when the drawn axis is perpendicular to the excitation light $\left(\vartheta=0^{\circ}, \gamma=90^{\circ}\right)$ (Figure 2). These two values are related to $\omega$ by the following equations,

$$
\begin{aligned}
& I_{\mathrm{V}}=I_{0}\left\langle\cos ^{2} \omega\right\rangle \\
& I_{H}=I_{0}\left\langle\sin ^{2} \omega\right\rangle \int_{0}^{2 \pi} \sin ^{2} \varphi \mathrm{d} \varphi / 2 \pi
\end{aligned}
$$

where, $\varphi$ is the angle as shown in Figure 5 . From these equations we can derive

$$
\left\langle\cos ^{2} \omega\right\rangle=I_{\mathrm{V}} /\left(I_{\mathrm{V}}+2 I_{\mathrm{H}}\right)
$$

The observed orientation factor $f$ is plotted against the $\%$-elongation of polymer films in Figure 6. Both deposited and doped films showed similar orientation behavior with the increase of elongation. The orientation in the surface region, which is shown by the orientation of deposited DPH, was a little higher than the average orientation in the whole film. In order to ensure these results, the orientation of surface and bulk was compared by other optical measurements. The birefringence was expressed by the differences $(\mathrm{d} n)$ between the refractive indices measured by the polarized 


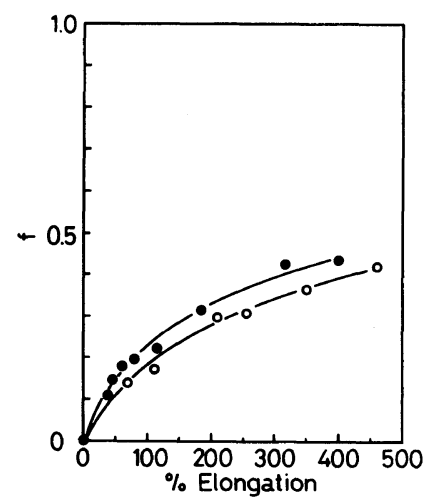

Figure 6. Orientation factor of drawn PVC films as a function of $\%$-elongation, measured by the fluorescence method. (O) DPH deposited PVC films, (O) DPH doped PVC films.

light vibrating in the direction of drawn axis and in the direction perpendicular to the drawn axis in the film surface. The orientation factor $f$ is derived from dividing $\mathrm{d} n$ by intrinsic birefringence $\mathrm{d} n_{0}$; this value was reported to be $10.4 \times 10^{-3}$ for PVC by Hibi et al. ${ }^{9}$

$$
f=\mathrm{d} n / \mathrm{d} n_{0}
$$

The birefringence of bulk was calculated from the retardation $r$ which was measured by a polarization microscope with a Berek compensator. The birefringence was derived by the following equation

$$
\mathrm{d} n=r / d
$$

where $d$ is the thickness of the polymer film measured by a micrometer. The birefringence of surface region was fundamentally measured by an Abbe refractometer, calibrated with a $\mathrm{Na}-\mathrm{D}$ line. The coordinate system abc, where $c$ is the drawn axis, is set as shown in Figure 7. The refractive indices, $n_{\mathrm{a}}\left(=n_{\mathrm{b}}\right)$ and $n_{\mathrm{c}}$, were measured using a polarizing filter equipped to the eyepiece of the refractometer. The birefringence $\mathrm{d} n$ is defined as,

$$
\mathrm{d} n=n_{\mathrm{c}}-n_{\mathrm{a}}
$$

The $\mathrm{d} n$ thus obtained gives the orientation factor $f$ for the surface region by eq 17 . The

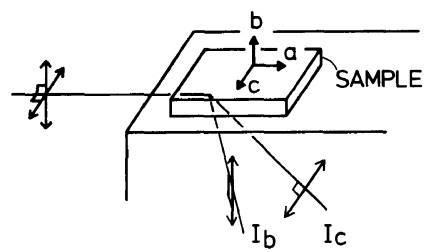

Figure 7. Setting of drawn PVC films on an Abbe refractometer; $c$ is the drawn axis.

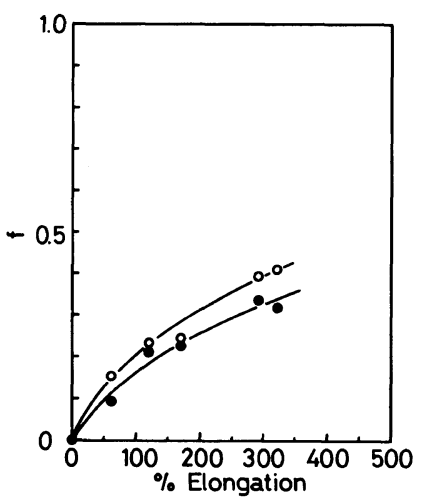

Figure 8. Orientation factor of drawn PVC films as a function of $\%$-elongation, measured from refractive indices. (O) surface, $(\bigcirc)$ bulk.

results are shown in Figure 8. Those results of birefringence measurements are similar to those of the fluorescence measurements, i.e., the surface orientation was slightly higher than the average. As the values of the birefringence were not large enough, the experimental errors of birefringence were somehow larger than those of the fluorescence.

\section{Orientation of Probes Deposited on the Oriented Surface}

The orientation of deposited probes on the PVC films which were drawn before deposition was compared with the films drawn after deposition. The orientation factor $f$ for the two kinds of DPH deposited systems was measured by the same apparatus in the previous section. The results are plotted against $\%$-elongation in Figure 9. The probes deposited on the drawn films showed higher orientation than the ones deposited before the drawing. This can be 


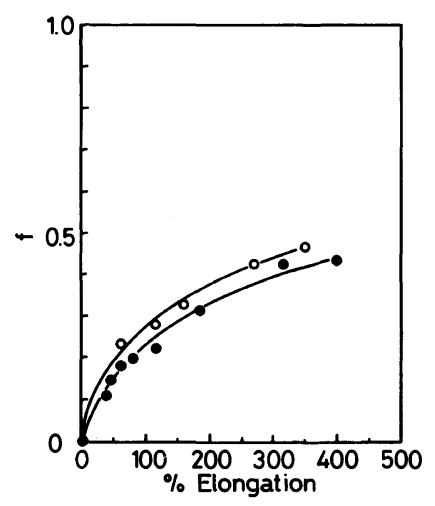

Figure 9. Orientation factor of DPH on drawn PVC films measured by the fluorescence method. (O) DPH was deposited on PVC film, followed by the drawing the film; (O) PVC film was drawn, followed by deposition of DPH on the film.

explained by considering that the fluorescent probes are preferentially introduced into the oriented region of the stretched samples. On the other hand, the fluorescent probes introduced before stretching showed the average orientation of the stretched samples. In this measurement, the fluorescence intensity from films deposited after drawing increased gradually with the increase of draw ratio. This increase on drawing was much greater than that calculated assuming the 3D-random orientation in the undrawn state and the constant deposition of the probes on the surface. In order to confirm this result, the absolute quantities of the deposited DPH were measured by dissolving the deposited films into THF, as mentioned above. The results are shown in Figure 10. As expected from the extraordinary increase of the total fluorescence intensity, larger quantities of DPH were observed to be deposited onto the elongated film compared with the undrawn one. This indicates that the rod-like probes tend to be preferentially adsorbed onto the oriented site of the polymer chain.

It is known that the bulk density of polymer films does not change so much on uniaxial stretching unless crystallinity changes drastically. The molecular motion of fluorescent

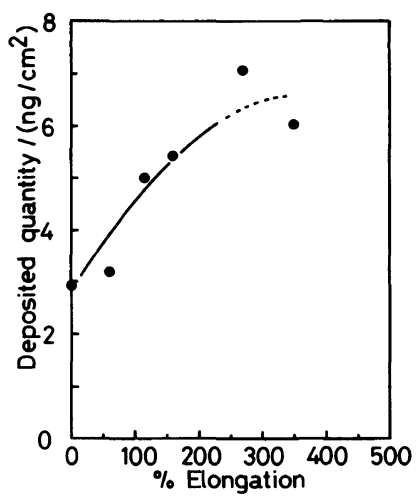

Figure 10. Deposited quantity of DPH as a function of $\%$-elongation.

probes in stretched polymer films has been studied in our laboratory by the fluorescence polarization method. When the polymer films were stretched, the polymer chain oriented to the stretching direction as did the anisotropic fluorescent probe. However, the molecular mobility of the probe within the lifetime of the fluorescence increased at the earlier stage of stretching in spite of the orientation. ${ }^{10}$ This means that there is an anisotropic structure of lower density in which fluorescent probes can easily enter the stretched polymer films. Though the local structure of the stretched polymer solid is not clearly understood yet, the present results provide some understanding of the local structure of a polymer solid.

\section{CONCLUSIONS}

The orientation of the probes deposited on the polymer surfaces, was measured sensitively by the fluorescence method. Moreover, the structure of the polymer film was scarcely disturbed by this deposition process of fluorescence probes. As a result of the application of the fluorescence method to PVC films, it was found that:

1) The DPH, a rod-like molecule, is deposited onto a polymer surface with 3Drandom orientation.

2) When the PVC is elongated at a tem- 
perature above $T_{\mathrm{g}}$, the orientation of surface is a little higher than that of the average.

3) DPH is deposited favorably onto the oriented site of a polymer chain.

These results show that this fluorescence polarization technique is quite useful for evaluating the surface structure of a polymer film.

\section{REFERENCES}

1. C. S. P. Sung, Macromolecules, 14, 591 (1981).

2. J. P. Hobbs, C. S. P. Sung, K. Krishnan, and S. Hill, Macromolecules, 16, 193 (1983).
3. F. M. Mirabella, J. Polym. Sci., Polym. Phys. Ed., 22, 1283 (1984).

4. F. M. Mirabella, J. Polym. Sci., Polym. Phys. Ed., 22, 1293 (1984).

5. L. H. Wang and R. S. Porter, J. Appl. Polym. Sci., 28, 1439 (1983).

6. Y. Nishijima, Y. Onogi, and T. Asai, J. Polym. Sci., C, 15, 237 (1966).

7. Y. Nishijima, J. Polym. Sci., C, 31, 353 (1970).

8. Y. Onogi, Doctoral Thesis, Department of Polymer Chemistry, Faculty of Engineering, Kyoto University (1977).

9. S. Hibi, M. Maeda, H. Kubota, and T. Miura, Polymer, 18, 137 (1977).

10. H. Tanaka, Y. Nishio, Y. Onogi, and Y. Nishijima, Rep. Prog. Polym. Phys. Jpn., 23, 563 (1980). 